\title{
Processo de Incorporação dos Inibidores de Protease para Hepatite C Crônica em Sistemas Públicos de Saúde: Estudo de caso comparativo entre Brasil, Austrália, Canadá e Inglaterra.
}

\section{Process of Incorporation and Guidance in Public Health Systems for Protease Inhibitors in Chronic Hepatitis C in: a comparative case study between Brazil, Australia, Canada and England.}

\section{Proceso de Incorporación de inhibidores de la proteasaen la hepatitis crónica $\mathrm{C}$ em Sistemas de SaludPública: unestudio de caso comparativo entre Brasil, Australia, Canadá e Inglaterra.}

Fernanda Dias DA SILVA ${ }^{1}$

Claudia Cristina de Aguiar PEREIRA ${ }^{2}$

Carla Jorge MACHADO ${ }^{3}$

RESUMO: Os estudos de Avaliação em Tecnologias em Saúde (ATS) subsidiam decisões quanto ao uso racional das tecnologias em saúde. Encontrar um equilíbrio entre a sustentabilidade dos sistemas de saúde, e o adequado acesso e utilização das tecnologias, representa um desafio e pressupõe o uso de ferramentas consistentes. Foram analisadas neste trabalho as características gerais das agências ou instituições responsáveis pela ATS, bem como o processo de avaliação e a incorporação dos inibidores de protease, telaprevir e boceprevir, para o tratamento da hepatite $\mathrm{C}$ crônica por meio de um estudo de caso comparativo de Brasil, Austrália, Canadá e Inglaterra. Trata-se de estudo de caso. Foi realizada pesquisa nos relatórios publicados sobre telaprevir e boceprevir, em 2013, nos endereços eletrônicos dos órgãos avaliadores dos países escolhidos. Observou-se, comparativamente a outras agências, que a Comissão brasileira (CONITEC), diferentemente das demais, aceitou a submissão e publicou no mesmo relatório a avaliação de medicamentos diferentes; sua composição tem perfil diverso das demais agências, cujos membros são indicados por sua expertise em ATS; o modelo de ATS é homogêneo entre os países; Houve divergências nos critérios de elegibilidade da populaçãoalvo, mesmo com aprovação do medicamento; o preço praticado no Brasil foi o menor; a Comissão 1 Escola Nacional de Saúde Pública Sergio Arouca - ENSP - Fundação Oswaldo Cruz - FIOCRUZ. Brasil. Especialista em Saúde Pública e Mestre em Ciências na área de Pesquisa Clínica em doenças infecciosas. E-mail: fernandadiassilva@gmail.com

2 Escola Nacional de Saúde Pública Sergio Arouca - ENSP - Fundação Oswaldo Cruz - FIOCRUZ. Graduação em Economia, mestrado em Demografia e doutorado em Population Health. E-mail:cpereira@ensp.fiocruz.br 3 Professora - Faculdade de Medicina - Universidade Federal de Minas Gerais. E-mail: carlajmachado@gmail.com 
Brasileira definiu no relatório de avaliação da incorporação do telaprevir e boceprevir também as regras de organização da rede assistencial, a elaboração de protocolos clínicos e diretrizes terapêuticas. Palavras-chave: Tecnologia Biomédica; Hepatite C; Inibidores de Protease

ABSTRACT: Health Technology Assessment (HTA) studies subsidize decisions regarding the rational use of health technologies. Finding a balance between the sustainability of health systems, and proper access and use of technology, is a challenge and requires the use of consistent tools. In this paper we analyzed the general characteristics of the agencies or institutions responsible for HTA in Brazil, Australia, Canada and England and we carried out a comparative case study to understand the incorporation of protease inhibitors telaprevir and boceprevir for the treatment of chronic hepatitis $\mathrm{C}$ in these countries. For that purpose, we used electronic published reports found on the websites of those HTA agencies regarding telaprevir and boceprevir in 2013. Compared to other agencies, the Brazilian Commission (CONITEC) unlike the others, accepted the submission and published a single report for both drugs; the HTA model was homogeneous across countries. There were differences in the eligibility criteria and target population, despite the fact of drug approval; the price charged in Brazil was the lowest; the Brazilian CONITEC also set the criteria for healthcare using the drugs and for clinical protocols and therapeutic guidelines. Keywords: BiomedicalTechnology; Hepatitis C; ProteaseInhibitors Keywords: Biomedical Technology; Hepatitis C; Protease Inhibitors

RESUMEN: Estudios de Evaluación de Tecnologías Sanitarias (ETS) pueden direccionar las decisiones relativas al uso racional de las tecnologías sanitarias. Encontrar un equilibrio entre la sostenibilidad de los sistemas de salud, y el acceso y el uso adecuado de la tecnología, es un reto y requiere el uso de herramientas consistentes. En este trabajo analizamos las características generales de los organismos o instituciones responsables de la HTA en Brasil, Australia, Canadá e Inglaterra y se realizó un estudio de caso comparativo de entender la incorporación de inhibidores de la proteasa telaprevir y boceprevir para el tratamiento de la hepatitis $\mathrm{C}$ crónica en estos países. Para ello, hemos utilizado los informes publicados electrónicos que se encuentran en los sitios web de esas agencias de ETS respecto a telaprevir y boceprevir en 2013. En comparación con otros organismos, la Comisión Brasileña (CONITEC) a diferencia de los otros, aceptó la presentación y publicamos un informe único para ambos fármacos; el modelo de HTA fue homogénea entre los países. Hubo diferencias en los criterios de elegibilidad y población objetivo, a pesar del hecho de la aprobación de medicamentos; el precio cobrado en Brasil fue la más baja; el CONITEC brasileño también establece los criterios para la asistencia sanitaria mediante las drogas y de los protocolos clínicos y directrices terapéuticas.

Palabras clave: Tecnología Biomédica; Hepatitis C; Inhibidores de la proteasa 


\section{INTRODUÇÃO}

A Avaliação de Tecnologia em Saúde (ATS) é um campo multidisciplinar da análise política que estuda de forma sistemática as implicações, diretas e indiretas, médicas, sociais, éticas e econômicas do desenvolvimento, difusão e uso das tecnologias em saúde ${ }^{1}$. Os estudos de ATS objetivam subsidiar a decisão quanto ao uso racional das tecnologias em saúde 2 .

Encontrar um equilíbrio entre a sustentabilidade dos sistemas de saúde, e oadequado acesso e utilização das tecnologias,representa um desafio e pressupõe o uso de ferramentas consistentes e capazes orientar a tomada de decisões.Grande parte das funções dos gestores de saúde consiste em selecionar uma combinação eficiente de tecnologias ${ }^{3}$.

O programa de ATS na Inglaterra foi estabelecido em 1993 como resultado de um comprometimento do sistema de saúde britânico (National Health System-NHS) em promover práticas baseadas em evidência. O National Institute for Healhand Care Excellence (NICE) foi criadoem 1999 e, a partir de 2005, além de efetuar a apreciação das tecnologias, ampliou suas funções, incluindo a avaliação e formulação de protocolos assistenciais ${ }^{4}$.

O primeiro programa de ATS no Canadá foi organizado em 1988,em Quebec, para auxiliar o Ministério da Saúde e do Serviço Social. Atualmente, a maioria das ATS é financiada pelo governo e desenvolvida por agências governamentais ${ }^{5}$.Em 1989, o CCOHTA (Canadian Coordination Office for Health Technology Assessment) começou a funcionaremtodo o paíse, em 2006, tornou-se o CADTH (Canadian Agency for Drugs and Technology in Health).

Existem, alémda CADTH, programas de ATS em Quebec (Institut National D'excellenceem Santé et en Services Sociaux (INESSS); Ontario (Medical Advisory Secretariat) e Alberta (Institute of Health Economics-IHE). Há pouca duplicidade de atividades entre eles e cada um dosprogramas adapta o conteúdo da análise no seu contexto jurisdicional ${ }^{5}$. No país, a economia resultante da utilização de ATS é estimada ente US\$ 16 mie US\$ 27 mi por ano ${ }^{5}$.

Na Austrália, cerca de $70 \%$ de todas as despesas em saúde são oriundas dofinanciamento público, sendo o governo nacional responsável por 70\% dos gastos. O Medicare, que subsidia os pagamentos aos médicos e profissionais de saúde associados, e o Pharmaceuticals Benefits Scheme (PBS), que auxilia os pagamentos dos medicamentos comprados em farmácias, são mantidos pelo governo australiano ${ }^{6}$.

No início dos anos 1970, foi desenvolvido um programa para avaliação da eficácia e segurança dos medicamentos financiados pelo PBS.AATS para medicamentos é realizada pelo Pharmaceutical Benefits Advisory Committee (PBAC), uma organização estatutária mantida pelo governo nacional. A PBAC é responsável por informar ao Ministério da Saúde quais medicamentos devem constar na lista do BPF. Este comitê analisa os dados de efetividade clínica, segurança e custo-efetividade². 
O Brasil, em 2002,iniciou o processo de institucionalização de ATS com a criação da Secretaria de Ciência Tecnologia e Insumos Estratégicos (SCTIE). A publicação da Lei n ${ }^{\circ}$ 2401/11 conferiu uma importância crescente ao processo de ATS na incorporação de novos medicamentos no País, especialmente àqueles de alto custo no âmbito do SUS.No mesmo ano, foi criada a Comissão Nacional de Incorporação de Tecnologias (CONITEC).

A CONITEC foi regulamentada pelo Decreto $n^{\circ} 7.646 / 11$ e é coordenada pela SCTIE,cujo papel éassessorar o Ministério da Saúde quanto à incorporação, exclusão ou alteração de novos medicamentos, produtos e procedimentos, bem como a constituição de protocolos clínicos e diretrizes terapêuticas.

A CONITEC tem o prazo de 270 dias, no máximo, para finalização da análise da proposta de incorporação feita pelos demandantes. Cabe à Secretaria Executiva, composta por uma equipe multidisciplinar, dar o apoio técnico e administrativo.A participação social do processo é legitimada pela Comissão Nacional de Saúde. A CONITEC é composta por 13 membros titulares, que representam diferentes órgãos, incluindo secretarias e agências do Ministério da Saúde, bem como dos seguintes conselhos: Conselho Nacional de Saúde (CNS); Conselho Nacional de Secretários em Saúde (CONASS); Conselho Nacional de Secretarias Municipais de Saúde (CONASEMS) e Conselho Federal de Medicina (CFM).

O produto da ATS realizadopela CONITEC é um relatório publicado em seu sítio eletrônico.

Telaprevir e boceprevirsão medicamentos novos com custos elevados para os sistemas de saúde, e cuja decisão de incorporação ocorreu recentemente no mundo e no Brasil através de ATS ${ }^{7,8}$. Esses medicamentos, que são inibidores de protease,têmação direta contra o vírus da hepatite $\mathrm{C}$ através do bloqueio da replicação do vírus. Os primeiros antivirais de ação direta são justamente o boceprevir e telaprevir. Ambos são utilizados com interferonpeguilado e ribavirina, constituindo uma terapia tripla. Os estudos mostraram que a adição dos IP ao tratamento padrão pode triplicar a chance de sucesso.Esses medicamentos foram registrados apenas para tratamento da hepatite $\mathrm{C}$ crônica pelo HCV genótipo 1.

O objetivo deste trabalho éanalisaras características gerais das agências ou instituições responsáveis pela ATS, bem como o processo de avaliação e a incorporação dos inibidores de protease, telaprevir e boceprevir, para o tratamento da hepatite $\mathrm{C}$ crônica por meio de um estudo de caso comparativo. Os países escolhidos para a análise comparativa com o Brasil foram Austrália, Canadá e Inglaterra, cujas instâncias avaliadoras são reconhecidas no cenário internacional pela competência e pioneirismo9. 


\section{MATERIAL E MÉTODOS}

Trata-se de estudo de caso. foi realizada pesquisa nos relatórios publicados sobre telaprevir e boceprevir, entre junho e agosto de 2013,nos endereços eletrônicos dos órgãos avaliadores dos países escolhidos: Nice (Reino Unido) ${ }^{10,11}$, CADTH (Canadá) ${ }^{12,13, ~ P B A C ~(A u s t r a ́ l i a) ~}{ }^{14,15}$ e CONITEC (Brasil) $)^{16}$, buscando o levantamento de uma série de critérios utilizados para incorporação ou adoção. para tanto, foramconstruídos dois quadros para análise comparativa do processo de incorporação ou adoção presentes nesses relatórios. as seguintes variáveis foram consideradas: : país; agência ou comitê avaliador; ano de criação da instituição da instância avaliadora; vínculo governamental (se há independência de unidade governamental e, se não há, com qualinstância avaliadora é vinculada); composição dos membros do comitê de avaliação; método de avaliação, ou seja, quais os tipos de estudos e opiniões que são considerados na avaliação; participação do público, ou seja, qual a forma de participação da sociedade para contribuir para a ats; se a decisão é de incorporação mandatória ou recomendatória; quaisosmedicamentos avaliados; proponente da incorporação dos medicamentos (organização ou laboratório interessado na avaliação);preços dos medicamentos, calculados com base nos dados da taxa cambial utilizados pela conitec, que correspondem à média geométrica da taxa de câmbio dos dois meses anteriores à avaliação que ocorreu em abril de 2012,ou seja, entre fevereiro e março de 2012 (para a libra esterlina, a taxa de câmbio utilizada foi de R\$2,75 e para o dólar americano, de R\$1,76); conteúdo da submissão, constituídas dos documentos necessários para que os avaliadores deliberem sobre a incorporação;conclusão, que trata da decisão das agências ou comitês após a avaliação;recomendações extras das agências ou comitês, data do relatório e de incorporação.além destas informações, no texto, se necessárias, outras são mencionadas.

\section{RESULTADOS}

Os dados referentes às agências/comissões de incorporação ou adoção de tecnologias em saúde, coletados nos relatórios publicados,foram dispostos no Quadro 1.

Quadro 1 - Matriz de comparação das Agências ou Comissões de Tecnologias em Saúde segundo Países selecionados, Agência, Vínculo Governamental, Composição do Comitê de Avaliação, Método de Avaliação, Participação Pública.

\begin{tabular}{|l|l|l|l|l|l|l|}
\hline País & $\begin{array}{l}\text { Agência } \\
\text { (sigla e data } \\
\text { de criação) }\end{array}$ & $\begin{array}{l}\text { Vínculo } \\
\text { Governa- } \\
\text { mental }\end{array}$ & $\begin{array}{l}\text { Composição } \\
\text { do Comitê de } \\
\text { Avaliação }\end{array}$ & $\begin{array}{l}\text { Método de } \\
\text { Avaliação }\end{array}$ & $\begin{array}{l}\text { Participação } \\
\text { Pública }\end{array}$ & $\begin{array}{l}\text { Incorporação/ } \\
\text { Adoção } \\
\text { mandatória }\end{array}$ \\
\hline Brasil & $\begin{array}{l}\text { CONITEC } \\
(2011)\end{array}$ & MS & $\begin{array}{l}13 \text { membros } \\
\text { definidos na } \\
\text { portaria (nomes } \\
\text { não mencionados } \\
\text { no relatório) e } \\
\text { consulta pública. }\end{array}$ & Não especificado & $\begin{array}{l}\text { Consulta pública por } \\
\text { 20 dias }\end{array}$ & Sim \\
\hline
\end{tabular}




\begin{tabular}{|c|c|c|c|c|c|c|}
\hline Inglaterra & NICE (1999) & $\begin{array}{l}\text { Independente, } \\
\text { mas financiada } \\
\text { pelo NHS }\end{array}$ & $\begin{array}{l}\text { Revisão de } \\
\text { evidência } \\
\text { pelo ERG. } \\
\text { Considerações } \\
\text { efetuadas } \\
\text { pelo NICE, } \\
\text { pelo Comitê } \\
\text { de avaliação } \\
\text { (indicado por } 3 \\
\text { anos), especialistas } \\
\text { e pacientes. }\end{array}$ & $\begin{array}{l}\text { São feitas } \\
\text { revisões e } \\
\text { críticas ao } \\
\text { dossiê. Análises } \\
\text { exploratórias } \\
\text { feitas pelo ERG. } \\
\text { O Comitê de } \\
\text { avaliação faz a } \\
\text { consideração das } \\
\text { evidências. }\end{array}$ & $\begin{array}{l}\text { Opinião } \\
\text { documentada dos } \\
\text { peritos clínicos e } \\
\text { dos pacientes não } \\
\text { relacionados ao } \\
\text { fabricante e nem ao } \\
\text { NICE }\end{array}$ & $\begin{array}{l}\text { Sim; abril } \\
\text { de } 2012 \text { para } \\
\text { ambos; } 90 \text { dias } \\
\text { de período para } \\
\text { adoção }\end{array}$ \\
\hline Canadá & $\begin{array}{l}\text { CADTH } \\
(1989)\end{array}$ & $\begin{array}{l}\text { Independente, } \\
\text { mas financiada } \\
\text { pelo governo } \\
\text { federal }\end{array}$ & $\begin{array}{l}14 \text { membros } \\
\text { indicados pelo } \\
\text { presidente da } \\
\text { CADTH, sendo } \\
2 \text { da sociedade } \\
\text { civil. Nomes são } \\
\text { mencionados no } \\
\text { relatório. }\end{array}$ & $\begin{array}{l}\text { Revisão dos } \\
\text { documentos } \\
\text { submetidos } \\
\text { e avaliação } \\
\text { das opiniões } \\
\text { de grupos de } \\
\text { pacientes pelo } \\
\text { CDEC }\end{array}$ & $\begin{array}{l}\text { Informação de } \\
\text { grupos de pacientes } \\
\text { sobre os desfechos } \\
\text { e outros assuntos } \\
\text { que são importantes } \\
\text { para os pacientes } \\
\text { são considerado na } \\
\text { recomendação da } \\
\text { CDEC }\end{array}$ & Não; \\
\hline Austrália & $\begin{array}{l}\text { PBAC } \\
(1984)\end{array}$ & $\begin{array}{l}\text { Independente, } \\
\text { mas financiada } \\
\text { pelo } \\
\text { Departamentof } \\
\text { Health } \\
\text { andAging }\end{array}$ & $\begin{array}{l}27 \text { membros } \\
\text { divididos em } \\
\text { dois subcomitês } \\
\text { (econômico e } \\
\text { clínico). Membros } \\
\text { indicados pelo } \\
\text { Governo. }\end{array}$ & $\begin{array}{l}\text { PBAC avalia os } \\
\text { estudos clínicos, } \\
\text { econômicos, } \\
\text { a população } \\
\text { estimada e } \\
\text { os impactos } \\
\text { financeiros. }\end{array}$ & $\begin{array}{l}\text { Comentários } \\
\text { enviados por } \\
\text { organizações e } \\
\text { associações médicas } \\
\text { são considerados. }\end{array}$ & Sim \\
\hline
\end{tabular}

Committee; CONITEC - Comissão Nacional de Criação de Tecnologias no SUS; ERG - Evidence Review Group MS - Ministério da Saúde; NHS - National Health System; NICE - National Institute for Health and Care; PBAC Pharmaceutical Benefits Advisory Committee

A CONITEC foi criada em 2011 e corresponde à instância avaliadora no Brasil e é a mais recente. A agência australiana foi criada em 1984, ou seja, vinte e sete anos antes da agência brasileira, sendo a mais a mais antiga.

Todos os países selecionados apresentavam a atividade de ATS institucionalizada, inclusive coma incorporação de novos medicamentos e outras tecnologias condicionadasà apreciação dos estudos clínicos e econômicos por estas agências ou comissões. As recomendações emitidas por tais instâncias são reconhecidas pelas autoridades de saúde como condição de incorporação ou rejeição. O Canadá constitui exceção, pois as recomendações da CADTH não representam, necessariamente, a decisão que o governo adotará.Observa-seainda que a comissão brasileira possui vínculo governamental, já as demais são independentes, mas financiadas por recursos do governo federal.

Os modelos de dossiê que devem ser submetidos são muito parecidos nos países analisados. Estes são compostos de uma revisão sistemática dos estudos clínicos e de uma análise que segue um modelo proposto pelas comissões ou agências. Todas as instâncias avaliadoras apresentam em seu endereço eletrônico um protocolo de como a análise econômica deve ser realizada.

A formação dos comitês de avaliação na Inglaterra, Canadá e Austrália era mais técnica, sendo grande parte de seus membros profissionais com atuação no campo de ATS e indicados pela agência ou governo. No Brasil, aplenário da CONITEC eracomposto por 13 integrantes, todos 
representantes dos órgãos e entidades definidos no Decreto ${ }^{\circ} 7446$.

A participação social foi claramente descrita nos relatórios emitidos pelo NICE, sendo citados os nomes dos representantes dos pacientes que são reconhecidos como 'pacientes especialistas'. A CADTH também descreveu as informações fornecidas pelos grupos de pacientes consultados em um tópico específico no relatório. $\mathrm{O}$ PABC não especificou a participação do público na avaliação publicada do boceprevir, mas mencionou associações médicas que participaram ou emitiram pareceres na avaliação descrita no relatório do telaprevir. A participação de pacientes não é mencionada. A CONITEC não descreve no relatório publicado sobre a participação do público ou de entidade médicas. No caso brasileiro, fazem parte da comissão um representante indicado pelo Conselho Federal de Medicina e um representante indicado pelo Conselho Nacional de Saúde. Além disso, no Brasil, o fluxo proposto para incorporação de um novo medicamento prevê consulta pública ou audiência pública anterior à decisão de incorporação, que cabe ao secretário da SCTIE. Os questionamentos discutidos na consulta pública não estão identificados no relatório final.

Especificamente quanto aos medicamentos (Quadro 2), os demandantes foram os fabricantes do boceprevir e telaprevir ${ }^{4}$, com exceção do Brasil,cuja interessada foi a Secretaria de Vigilância em Saúde (SVS)para os dois inibidores de protease e a Merck Sharp \&Dhome (MSD), fabricante do boceprevir. A empresa fabricante do telaprevir, Janssen, não foi descrita como demandante.

Quadro 2 - Matriz de comparação das Agências ou Comissões de Tecnologias em Saúde quanto ao boceprevir e telaprevir segundo Países selecionados.Dados sobre proponentes, preço, conteúdo da submissão, conclusão e recomendações extras do parecer, data do relatório, período para incorporação da tecnologia.

(continua)

\begin{tabular}{|l|l|l|l|l|l|}
\hline País & Proponentes & Preço & $\begin{array}{l}\text { Conteúdo da } \\
\text { Submissão }\end{array}$ & $\begin{array}{l}\text { Conclusão } \\
\text { do } \\
\text { Parecer }\end{array}$ & $\begin{array}{l}\text { Recomendações } \\
\text { extras; data do } \\
\text { relatório; período } \\
\text { para incorporação } \\
\text { da ATS }\end{array}$ \\
\hline Brasil & SVS e MSD (BOC) & $\begin{array}{l}\text { R\$ } \\
48.798,57 \\
(\mathrm{BOC}) \\
\text { e R\$ } \\
57.639,74 \\
(\mathrm{TVR})\end{array}$ & $\begin{array}{l}1 \text { dossiê contendo revisão } \\
\text { sistemática, análise } \\
\text { econômica e impacto } \\
\text { orçamentário. Dados } \\
\text { disponibilizados em sítio } \\
\text { eletrônico. }\end{array}$ & $\begin{array}{l}\text { Deferido para pacientes } \\
\text { com hepatite crônica } \\
\text { genótipo 1 F3 ou F4. } \\
\text { Exclui coinfectados } \\
\text { com HIV ou HBV. TVR } \\
\text { como escolha para nulos } \\
\text { F4. Propõe redução de } \\
\text { preços em 30\%. }\end{array}$ & $\begin{array}{l}\text { Solicita estudo } \\
\text { observacional } \\
\text { e estabelece } \\
\text { protocolo clínico } \\
\text { e reorganiza } \\
\text { rede para } \\
\text { implementação; } \\
\text { abril de 2012; 180 } \\
\text { dias prorrogável } \\
\text { por mais } 90 .\end{array}$ \\
\hline
\end{tabular}

4 Brasil: MSD; Inglaterra: Janssen e MSD; Canadá: VertexPharmaceuticals Inc. e Merck Canada Inc; Austrália: Janssen-Cilag PtyLtd. E MSD PtyLtd. 


\begin{tabular}{|c|c|c|c|c|c|}
\hline Inglaterra & $\begin{array}{l}\text { TVR: Janssen; } \\
\text { BOC: MSD }\end{array}$ & $\begin{array}{l}£ 22.398 \text { ou } \\
\mathrm{R} \$ 61.594 \\
(\mathrm{BOC}) \mathrm{e} £ \\
30.800 \mathrm{ou} \\
\mathrm{R} \$ 84.700 \\
(\mathrm{TVR})\end{array}$ & $\begin{array}{l}\text { TVR: } 1 \text { dossiê do } \\
\text { fabricante com revisão } \\
\text { sistemática de } 6 \text { ECRe } \\
\text { de } 6 \text { estudos de custo } \\
\text { efetividade e análise } \\
\text { econômica pela } \\
\text { perspectiva do NHS e da } \\
\text { sociedade; BOC: } 1 \text { dossiê } \\
\text { preparado pelo fabricante } \\
\text { com } 5 \text { ECR e } 1 \text { ECR fase } \\
\text { II, análise econômica pela } \\
\text { perspectiva do NHS e da } \\
\text { sociedade }\end{array}$ & $\begin{array}{l}\text { TVR ou BOC: Deferido } \\
\text { para pacientes com } \\
\text { hepatite crônica genótipo } \\
1 \text { tratados previamente } \\
\text { ou não. Mesmo não } \\
\text { representados nos } \\
\text { estudos, parecer } \\
\text { favorável à inclusão de } \\
\text { co-infectados e usuários } \\
\text { de drogas. }\end{array}$ & $\begin{array}{l}\text { Não há; abril de } \\
2012 \text { para ambos; } \\
90 \text { dias }\end{array}$ \\
\hline
\end{tabular}

Notas: TVR - telaprevir: BOC - boceprevir; MSD - Merck Sharp \&Dhome; ECR - ensaio clínico randomizado; NHS - National Health System

Quadro 2 - Matriz de comparação das Agências ou Comissões de Tecnologias em Saúde quanto ao boceprevir e telaprevir segundo Países selecionados. Dados sobre proponentes, preço, conteúdo da submissão, conclusão e recomendações extras do parecer, data do relatório, período para incorporação da tecnologia.

(conclusão)

\begin{tabular}{|c|c|c|c|c|c|}
\hline País & Proponentes & Preço & $\begin{array}{l}\text { Conteúdo da } \\
\text { Submissão }\end{array}$ & $\begin{array}{l}\text { Conclusão } \\
\text { do } \\
\text { Parecer }\end{array}$ & $\begin{array}{l}\text { Recomendações } \\
\text { extras; data do } \\
\text { relatório; período } \\
\text { para incorporação } \\
\text { da ATS }\end{array}$ \\
\hline Canadá & $\begin{array}{l}\text { TVR: } \\
\text { VertexPharmaceutical } \\
\text { Inc.; BOC: Merck } \\
\text { Canada Inc. }\end{array}$ & $\begin{array}{l}£ 34.968 \text { ou R\$ } \\
61.544(\mathrm{BOC}) \mathrm{e} \\
£ 46.200 \mathrm{ou} \mathrm{R} \$ \\
81.312(\mathrm{TVR})\end{array}$ & $\begin{array}{l}\text { TVR: Revisão } \\
\text { sistemática de } 5 \\
\text { estudos clínicos } \\
\text { quanto à efetividade } \\
\text { ou eficácia, segurança } \\
\text { do medicamento e } \\
\text { revisão da análise de } \\
\text { custo-efetividade; } \\
\text { BOC: Revisão } \\
\text { sistemática de } 3 \\
\text { estudos clínicos } \\
\text { quanto à efetividade } \\
\text { ou eficácia, segurança } \\
\text { do medicamento e } \\
\text { revisão da análise de } \\
\text { custo-efetividade. }\end{array}$ & $\begin{array}{l}\text { TVR ou BOC: O } \\
\text { parecer é favorável } \\
\text { à inclusão para } \\
\text { pacientes com HCV } \\
\text { verificado por RNA } \\
\text { com validade de } 6 \\
\text { meses, F2, F3 ou } \\
\text { F4. É favorável à } \\
\text { exclusão de pacientes } \\
\text { coinfectados com } \\
\text { HIV. }\end{array}$ & $\begin{array}{l}\text { Sugere redução do } \\
\text { preço e apenas } 1 \\
\text { curso de tratamento, } \\
\text { e prioridade para } \\
\text { pacientes com } \\
\text { escores mais } \\
\text { avançados de fibrose; } \\
\text { janeiro de } 2012 \text { para } \\
\text { TVR e setembro de } \\
2011 \text { para BOC; não } \\
\text { especificado }\end{array}$ \\
\hline Austrália & $\begin{array}{l}\text { TVR: Janssen-Cilag } \\
\text { PtyLtd; BOC: MSD } \\
\text { (Australia) PtyLtd }\end{array}$ & $\begin{array}{l}\text { Não } \\
\text { especificado }\end{array}$ & $\begin{array}{l}\text { TVR: } 1 \text { documento } \\
\text { elaborado com } 4 \\
\text { ECR; } 1 \text { avaliação } \\
\text { econômica de custo } \\
\text { utilidade. BOC: } 1 \\
\text { documento elaborado } \\
\text { com } 2 \text { ECR; } 1 \\
\text { avaliação econômica } \\
\text { de custo utilidade. }\end{array}$ & $\begin{array}{l}\text { TVR ou BOC: } \\
\text { Conclui pelo } \\
\text { deferimento para } \\
\text { pacientes com HCV } \\
\text { genótipo } 1, \text { maiores } \\
\text { de } 18 \text { anos. }\end{array}$ & $\begin{array}{l}\text { Sugere redução de } \\
\text { preço; julho de } 2012 \\
\text { para ambos; não } \\
\text { especificado }\end{array}$ \\
\hline
\end{tabular}

Notas: TVR - telaprevir: BOC - boceprevir; MSD - Merck Sharp \&Dhome; ECR - ensaio clínico randomizado; NHS - National Health System 
Os preços dos medicamentos praticados no Brasil foram menores do que nos países estudados. A Austrália não especificou os preços.

A submissão e a avaliaçãoforam feitas de forma conjunta para o boceprevir e telaprevir apenas para CONITEC. Para as demais instâncias avaliadoras, cada um dos medicamentos foi submetido e avaliado separadamente.

As recomendações foram todas publicadas em formato de relatório, cujas versões - resumida ou integral - podem ser acessadas através do endereço eletrônico dos órgãos de avaliação ${ }^{10-16}$. Apenas nos relatórios publicados pela CADTH e pelo NICE,a relação nominal dos profissionais que avaliaram a inclusão do telaprevir e boceprevirestãodescritas nos relatórios.

Todos os órgãos de avaliação recomendaram a inclusão de boceprevir e telaprevir para o tratamento de hepatite $\mathrm{C}$ crônica, genótipo 1. O perfil dos pacientes elegíveis para o tratamento com esses medicamentos variou entre os países. A CONITEC foi a instância que apresentou mais restrições. Para a CONITEC, o tratamento com inibidores da protease deveria ser restrito aos pacientes com estádio de fibrose classificados como F3 ou F4 (escore METAVIR) e em pacientes não coinfectados com vírus HIV.

A recomendação final emitida pelo CADTH foi presença de fibrose maior ou igual a F2 (escore METAVIR) em pacientes não coinfectados.

O relatório publicado pelo PBCA considerou como critério de elegibilidade a confirmação de hepatite C crônica e idade superior a 18 anos. Esses medicamentos foram incluídos na lista do PBS em uma categoria que prevê utilização em unidades hospitalares que preencham alguns critérios de qualidade e assistência previamente estabelecidos.

A CONITEC, além do parecer final que recomendou a incorporação, vinculou a avaliação de incorporação desses medicamentosà autorização centralizada para controle das prescrições nos comitês estaduais; ao estabelecimento de fluxos para disponibilização dos medicamentos; à definição do perfil dos serviços públicos que possuem experiência no tratamento da hepatite C; ao desenvolvimento de um estudo observacional para avaliação da efetividade e segurança do medicamento num cenário de vida real.

O NICE decidiu pela adoçãodosmedicamentos para o tratamento de pacientes portadores de hepatite $\mathrm{C}$ crônica e não estabeleceu outros critérios para elegibilidade dos pacientes.Foi citada no relatório a inexistência de razões para exclusão de portadores de coinfecção por HIV ou usuários de drogas injetáveis. 


\section{DISCUSSÃO}

O presente estudo de caso comparou, além de características gerais das agências ou instâncias avaliadoras de tecnologias em saúde,relatórios publicados sobre a avaliação para incorporação de medicamentos utilizados para o tratamento da hepatite $\mathrm{C}$ crônica - telaprevir e boceprevir. Foram utilizadas informações do Brasil, Inglaterra, Canadá e Austrália.

A avaliação de tecnologias em saúde (ATS) é utilizada em países desenvolvidos como um subsídio importante para as decisões sobre cobertura de tecnologias/procedimentos e para elaboração de diretrizes clínicas, e, portanto, para os processos de planejamento e avaliação de serviços e programas de saúde ${ }^{10}$. Dessa forma, compreender se o Brasil se encontra em patamar similar a esses países no que se refere às características gerais dos procedimentos de ATS nesses países e à avaliação de medicamentos específicos tornou-se importante.

A comparação dos preços praticados desses medicamentos mostrou que, no Brasil, os valores foram inferiores àqueles dos demais países. Segundo o relatório da CONITEC, a Câmara de Regulação do Mercado de Medicamentos (CMED) pode ter tido um importante papel na redução dos preços aplicados no Brasil. Esse dado aponta que, talvez, seja possível uma redução dos valores nos demais países. O CADTH recomendou a redução de preço como uma importante medida em seu relatório.

A forma como a CONITEC é composta também a torna diferente das demais. No Brasil, os membros são escolhidos de acordo com a posição hierárquica no Ministério da Saúde ou nas organizações vinculadas. A formação está definida no Decreto $n^{\circ} 7646$, de 21 de dezembro de 2011, e na Portaria ${ }^{\circ} 204$, de 7 de fevereiro de 2012. Nos demais países analisados, a composição é baseada no perfil profissional de cada membro que deve ter expertise em ATS. Seria desejável que, no Brasil, sempre que possível, os membros do comitê fossem escolhidos pela afinidade com o tema e pela expertise específica na ATS. Assim, uma questão correlata é o grau de independência da análise realizada, uma vez que alguns membros são subordinados ao Ministério da Saúde. As demais agências, apesar de serem financiadas pela autoridade sanitária de cada país, têm uma composição baseada nas capacidades acadêmicas e profissionais e não em posições hierárquicas ${ }^{11}$.

A CONITEC decidiu pela incorporação dos inibidores de protease, boceprevir e telaprevir, mas estabeleceu critérios de elegibilidade para o tratamento baseado no grau de comprometimento hepático verificado na biópsia. Foram considerados como candidatos à utilização desses medicamentos pacientes com escore METAVIR maior ou igual a F3. A exclusão do grupo de pacientes com escore METAVIR menor que F3 foi justificada pela afirmação de que novos medicamentos estão sendo estudados e que esse grupo poderia esperar em virtude da progressão lenta da doença. A exclusão de pacientes coinfectados não foi claramente justificada, mas sabe-se que tal grupo de pacientes foi excluído dos estudos clínicos utilizados na revisão sistemática feita para comprovação de eficácia. Cabe ressaltar que o tratamento da hepatite $\mathrm{C}$ crônica, no Brasil, foi 
regulamentado pelo Protocolo Clínico e Diretrizes Terapêuticas (PCDT) para Hepatite Viral C e Coinfecções, publicado em 2011, antes da incorporação do telaprevir e boceprevir,e que também estabelecia critérios de elegibilidade para o tratamento, além da confirmação de hepatite $\mathrm{C}$ crônica. Uma das condições era a lesão hepática confirmada por biópsia hepática de escore METAVIR igual ou maior a F2, ou maior ou igual a F1 na presença de atividade inflamatória. Esse protocolo continua vigente. Com a incorporação dos inibidores de protease, foi publicado um suplemento ao PCDT, de forma que existem tratamentos diferentes de acordo com o grau de lesão hepática apresentada pelos pacientes. O fato é que a justificativa alegada de que os pacientes poderiam esperar por medicamentos mais seguros não parece razoável, visto que os pacientes com escore METAVIR menor que F3 são elegíveis para o tratamento com ribavirina e interferonpeguilado, menos eficazes do que boceprevir e telaprevir. A justificativa que, talvez, fosse adequada, seria que, para um grupo de portadores, cuja lesão hepática não é avançada, o tratamento com telaprevir ou boceprevirteria menor custo-efetividade e/ou impactos financeiros maiores.

A agência canadense justificou a restrição quando oescore METAVIR fosse maior ou igual a F2, argumentando que a hepatite $\mathrm{C}$ crônica é uma doença de progressão incerta, pois muitos pacientes não irão progredir para uma doença hepática progressiva, e que o tratamento tem um potencial de dano. Foi considerado que o alto custo desses medicamentos, e a estimativa de custo-efetividade,foi menos favorável em pacientes com fibrose menos avançada. A justificativa para exclusão dos pacientes coinfectados com HIV foi baseada em que os benefícios de boceprevir ou telaprevir nesse grupo de pacientes não estava claro, poistais pacientes foram excluídos dos estudos utilizados na revisão sistemática para comprovação de eficácia. A questão da interação medicamentosa desses remédioscom os antirretrovirais poderia implicar ajustes de doses do telaprevir ou boceprevir. Cabe ressaltar que a mudança de tratamento para hepatite $\mathrm{C}$ crônica afetou todos os pacientes elegíveis para tratamento.

Apesar dos inibidores de protease terem sido aprovados para a lista do Pharmaceutical Benefits Scheme (PBS), as avaliações publicadas anteriormente pelo Pharmaceutical Benefits Advisory Committee (PBAC) rejeitaram a incorporação desses medicamentos. Na ocasião, os relatórios justificaram a rejeição, pois os medicamentos não haviam sido aprovados pela regulação e, portanto, havia incerteza sobre os critérios de utilização dos medicamentos, o que poderia alterar a análise de custo-efetividade. Os fabricantes fizeram uma nova submissão após a decisão da regulação, e os medicamentos foram incluídos na lista do PBS dentro de uma categoria que prevê utilização em unidades hospitalares.

O NationalInstitute for Health and Care (NICE) foi a instância avaliadora que menos restrições apresentou ao uso dos medicamentos. Uma das alegações dos pacientes era a presença do estigma associado à infecção por HCV por sua associação com uso de drogas injetáveis. O NICE também considerou que a queda na prevalência do HCV poderia resultar em queda na incidência, o que seria um ganho em saúde pública. 
Além da definição dos critérios de elegibilidade para os portadores de hepatite $\mathrm{C}$ crônica, a CONITEC estabeleceu condições de utilização e acesso ao novo tratamento. Assim, as considerações empregadas no relatório de avaliação publicado pelo plenário da CONITEC extrapolaram uma mera avaliação de incorporação de tecnologia, elaborando em seu parecer diretrizes clínicas e um protocolo clínico. Apesar de ser competência da CONITEC, a ATS e a elaboração de protocolos clínicos e diretrizes terapêuticas são atividades distintas.Nos demais países analisados, as restrições impostas ao uso dos medicamentos seguiram uma razão técnica e não ultrapassaram as competências de uma agência/comitê de avaliação de tecnologias. Na Austrália, a organização da assistência farmacêutica classifica os medicamentos de acordo com seu perfil dentro de algumas categorias. O telaprevir e boceprevir foram categorizados como highly specialised drug program (programa de medicamentos altamente especializados; tradução livre), que apresenta regras próprias de utilização. A questão é que, no Brasil, essas regras foram criadas no relatório de ATS, mesmo existindo no país uma orientação da assistência farmacêutica que definiua segmentação dos medicamentos de acordo com a lógica de distribuição e financiamento em componente básico, estratégico ou especializado, tendo cada um deles regras próprias. O que foi recomendado pela CONITEC e determinado Nota Técnica Conjunta n01/2013 - DDAHV/SVS/MS e DAF/SCTIE/ MS é absolutamente específico para os medicamentos boceprevir e telaprevir, que propôs uma reorganização de serviços já estabelecidos. A criação de uma nova categoria para tais medicamentos retardou o acesso da população à nova tecnologia.

Esses medicamentos foram aprovados em abril de 2012 e, conforme a previsão legal, teriam, no máximo, 270 dias (dezembro de 2012) para estarem disponíveis à população. Até maio de 2013, nenhum paciente havia iniciado o tratamento com esses medicamentos no SUS por vias regulares. Os medicamentos foram comprados de forma centralizada, mas a efetiva dispensação deles foi prejudicada pelas regras impostas na nota técnica $n^{\circ} 01 / 2013$ - DDAHV/SVS/MS e DAF/SCTIE/ MS para inibidores de protease no tratamento de hepatite $\mathrm{C}$ crônica. Cabe ressaltar que o elenco de medicamentos disponíveis no SUS para tratamento da hepatite $\mathrm{C}$ crônica estão incluídos dentro do componente especializado da assistência farmacêutica e seguem as regras de financiamento e dispensação desse grupo de insumos. No entanto, para os IP, outras regras de dispensação foram formuladas, tal que praticamente uma nova categoria foi criada exclusivamente para o boceprevir e telaprevir.

O atraso na utilização desses medicamentos, mesmo já adquiridos pelo governo, pode estar relacionado às barreiras impostas pelas normas de incorporação da CONITEC e pelas regras de utilização definidas na nota técnica. A adoção desses preceitos pode trazer como consequência iniquidade no acesso, através da utilização por parcela da sociedade em ações judiciais que, em geral, não contemplam os parâmetros técnicos de uso de medicamentos. 


\section{CONCLUSÃO}

Conhecer como a avaliação e incorporação de tecnologias são realizadas no Brasil é de grande interesse, sobretudo no sentido de promover que os princípios de universalidade, integralidade e equidade sejam orientadores dessa atividade.

A publicação da lei $\mathrm{n}^{\circ} 12.401$, de 28 de abril de 2011, definiu o conceito de assistência terapêutica integral e determinou como atribuição do Ministério da Saúde a incorporação, exclusão e alteração pelo SUS de novos medicamentos de forma assessorada pela CONITEC. Essa instância avaliadora ligada ao MS passou a exercer um papel relevante no âmbito nacional.

Observaram-se, comparativamente a outras agências, as seguintes semelhanças e diferenças: a CONITEC, diferentemente das demais instâncias, aceitou a submissão e publicou no mesmo relatório a avaliação de medicamentos diferentes; a composição da CONITEC tem um perfil diverso das demais agências, cujos membros são indicados por sua expertise em ATS e, de fato, na CONITEC, a participação dos membros tem um cunho comparativamente mais político; o modelo de ATS (eficácia clínica e avaliação econômica) é homogêneo entre as agências/comissões; a aprovação desses medicamentos foi unânime nos países estudados, apesar de divergências nos critérios de elegibilidade da população-alvo; a incorporação foi feita praticamente na mesma época em todos os países; o preço praticado no Brasil é inferior aos praticados nos demais países estudados; a CONITEC definiu em seu relatório de avaliação sobre a incorporação do telaprevir e boceprevir também as regras de organização da rede assistencial.

\section{REFERÊNCIAS BIBLIOGRÁFICAS}

1. Kristensen FB, Lampe K, Chase DL, Lee-Robin SH, Wild C, Moharra M, et al. Practical tools and methods for health technology assessment in Europe: structures, methodologies, and tools developed by the European Network for Health Technology Assessment, EUnetHTA. Int J Technol Assess Health Care. 2009; 25 Suppl 2:1-8.

2. Cuyler AJ. Four issues in cost-efectiveness analysis and health technology assessment: a view from the touch-line. In: Llano Señaris JE, Campillo-Artero C. Health Technology Assessment and Health Policy Today: a multifaceted view of the unstable crossroads. Switlzerlad: Springer International Publishing; 2015. p. 77-94.

3. Silva EN, Silva MT, Elias FTS. Sistemas de Saúde e Avaliação de Tecnologias em Saúde. Em: Nita ME, Secoli SR, Nobre MRC, editores. Avaliação de Tecnologias em Saúde: evidência clínica, análise econômica e análise de decisão. Porto Alegre: Artmed; 2010. p. 419-32.

4. Polanczyk CA, Vanni T, Kuchenbecker RS. Avaliação de Tecnologias em Saúde no Brasil e no Contexto Intenacional. Em: Nita ME, Secoli SR, Nobre MRC, editores. Avaliação de Tecnologias em Saúde: evidência clínica, análise econômica e análise de decisão. Artmed; 2010. p. 433-49. 
5. Hailey DM. Health technology assessment in Canada: diversity and evolution. Med J Aust. 2007;187(5):286.

6. Hailey D. The history of health technology assessment in Australia. Int J Technol Assess Health Care. 2009; 25(S1):61.

7. Morihara D, Watanabe H, Takata K, Iwashita H, Tsuchiya N, Kunimoto H, et al. Adjusting the starting dose of telaprevir according to renal function decreases adverse effects and affects the sustained virological response rates. Eur J GastroenterolHepatol. 2015; 27(1): 55-64.

8. Hutchison JG, Manns MP, Muir JA, Terrault NA, Jacobson IM, Afdhal NH, et al. Telaprevir for previously treated chronic HCV infection. N Eng J Med 2010; 362: 1292-1303.

9. Wilsdon T,Fiz E,Haderi A. A comparative analysis of the role and impact of Health Technology Assessment 2013 (Final Report). [Internet] Brussels: Charles River Associate. May 2014 [citado em 2015 Feb 01]. Disponível em: http://www.efpia.eu/uploads/documents/cracomparative-analysis.pdf

10. National Institute for Health and Care Excellence. Telaprevir for the treatment of genotype 1 chronic hepatitis C (technology appraisal guidance TA 252). Londres: April 2012 [citado em 2015 Feb 01].Disponível em:https://www.nice.org.uk/guidance/ta252

11. National Institute for Health and Care Excellence. Boceprevirfor the treatment of genotype 1 chronic hepatitis C (technology appraisal guidance TA 253). Londres: April 2012 [citado em 2015 Feb 01]. Disponível em: https://www.nice.org.uk/guidance/ta253

12. Canadian Agency for Drugs and Technology in Health. CDEC Final Recommendation: Telaprevir. Toronto: June 2013 [citado em 2015 Feb 01]. Disponível em: http://www.cadth.ca/ media/cdr/complete/cdr complete Incivek\%20RFA-SR0311-June-14-13.pdf

13. Canadian Agency for Drugs and Technology in Health. CDEC Final Recommendation: Boceprevir. Toronto: June 2013 [citado em 2015 Feb 01]. Disponível em: http://www.cadth.ca/ media/cdr/complete/cdr_complete_SF0312-Victrelis_RFA June-14-13.pdf

14. Pharmaceutical Benefits Advisory Committee (PBAC). PublicSummaryDocument: Telaprevir. Sydney: June 2012 [citado em 2015 Feb 01]. Disponível em: http://www.pbs.gov.au/ industry/listing/elements/pbac-meetings/psd/2012-07/telaprevir.pdf

15. Pharmaceutical Benefits Advisory Committee (PBAC). PublicSummaryDocument: Boceprevir. Sydney: June 2012 [citado em 2015 Feb 01]. Disponível em: http://www.pbs.gov.au/ industry/listing/elements/pbac-meetings/psd/2012-07/boceprevir.pdf

16. Ministério da Saúde. Secretaria de Ciência, Tecnologia e Insumos Estratégicos. Inibidores de protease (Boceprevir e Telaprevir) para o tratamento da hepatite crônica C. Brasília: July 2012 [citado em 2015 Feb 01]. Disponível em: http://conitec.gov.br/images/Relatorios/2012/ Boceprevir_Telaprevir_final.pdf

17. Krauss-Silva L. Avaliação tecnológica em saúde: questões metodológicas e operacionais 
Technology assessment in healthcare: methodologicalandoperationalquestions. Cad Saúde Pública. 2004;20(Sup 2):S199-S207.

18. Brasil, Secretaria de Ciência T e IE, Departamento de Ciência e Tecnologia. Política nacional de gestão de tecnologias em saúde. Brasília, DF: Ministério da Saúde; 2010.

Artigo apresentado em 25-06-14 Artigo aprovado em 06-07-15 Artigo publicado no sistema em 26-09-15 